\title{
A Brief Introduction to Ansoffian Theory and the Optimal Strategic Performance-positioning Matrix on Small Business (OSPP)
}

\author{
John F. Gianos
}

Received: February 29, 2012

Accepted: January 24, 2013 Published: April 1, 2013

doi:10.5296/jmr.v5i2.3129

URL: http://dx.doi.org/10.5296/jmr.v5i2.3129

\begin{abstract}
The purpose of this paper is to briefly introduce the reader to Igor Ansoff and briefly examine the praxis of the components of Ansoff's Strategic Success Paradigm which when implemented have proven empirically to increase the firm's probability of strategic success.. We will look at efficacy of use of each of its components relative to the formulation and implementation of strategy in a for-profit Small sized enterprise. coalesced with other modeling techniques like Porter's, Strengths, Weaknesses, Opportunities, Threats (SWOT), Ansoff's strategic diagnosis with the Optimal Strategic Performance Position (OSPP) tool can provide management with an enriched capability to evaluate the firm's current and future performance position providing both a descriptive plan as well as a prescriptive diagnosis
\end{abstract}

Keywords: Ansoff, Strategy, Environmental Turbulence, Strategic Success Paradigm, Optimal Strategic Performance Position (OSPP) tool 


\section{Introduction}

There is a recognized need to have a consistent and comprehensive framework to analyze a Small Business's (SB) strategic posture. Conduct a strategic gap analysis to identify where are company's shortfalls in capability can be important. Using the Optimal Strategic Performance Position (OSPP) tool in order to ascertain the competitive positioning of a small SB may assist in company ascertaining its gaps in capability. Using this tool and Ansoff's model may yield a descriptive and prescriptive plan that enables the managers of a small business to optimize the firm's performance.

Thus far, most research on Ansoff's approach to strategic positioning has been focused on large and medium sized organizations. This article will focus on a small business as defined by SBA. Even though this article will be conceptual, it will endeavor to present a real life, robust model combining Ansoff's strategic diagnosis with elements of the performance matrices to provide the SB management with an enhanced capability to evaluate a firm's current and future performance position.

The purpose of this paper is to examine Ansoff's theories and to briefly examine the Optimal Strategic Performance Position (OSPP) tool that may be used by management analyze its strategic posture. Discuss how Ansoff's contributions may enhance a businesses performance through the analysis of an industries environment turbulence level relative to its aggressiveness, responsiveness of its capability or Strategic Success Paradigm (SSP). The OSPP tool asserts that it allows a manager to assess their business and its different variables mathematically to match the current and future environment of its industry by testing the SSP factors.

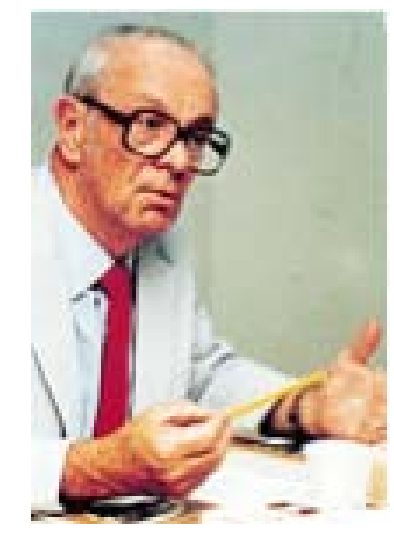

H. Igor r Ansoff (1918-2002) was an applied mathematician and business manager. He is well known as the pioneer and has been declared by some to be the father of Strategic Management. Although Russian born, Ansoff studied at Brown University, where he received his Doctorate in applied mathematics; his mathematical expertise served as the basis for his analysis of strategic management techniques. Ansoff's career was quite varied ranging from academia to the US Navy and engineering departments at private sector firms (El-Kadi Consulting, n.d.) . In 1950 he worked at the prestigious think tank, RAND. While at RAND 
he focused on developing solutions for NATO's strategic challenges; this work became the underpinning for his theories on strategic management (The Economist, 2008). At the end of his career, Ansoff was a distinguished professor at United States International University (now Alliant International University) where his work in strategic management research is still continued

Ansoff introduced the concept of balancing "external characteristics of the product-market strategy and [creating] internal fit between strategy and business resources” (Ansoff, 2007). Ansoff's work is based on developing an instrument which facilitates a top manager's ability to analyze data with the objective of exploring and exploiting the "future profit potential" and, as a result, improve the firm's competitive position. Ansoff's approach can quantify information in a way that enables management to match their behavior and capabilities to the external operating environment. He noted that managers frequently try a "one-size fits all" approach and do not vary their plans and behaviors based on current conditions; instead they tend to develop plans and manage in ways that are based only on historical data. Ansoff was able to empirically prove that using data to account for both historical and future scenarios as well as changing plans and behavior to match these scenarios as they evolve is a valid method for optimizing the firm's success (Ansoff, 2007).

We will discuss Ansoff's Strategic Success Paradigm and principles for use by Small and Medium Sized Enterprises, which when implemented have proven empirically to increase the firm's probability of strategic success. There is also strong empirical support linking a positive causal relationship between formalized strategic planning and achieving optimal financial success of a business organization (Ansoff, Brandenburg, and Radosevich. 1971)

Ansoff divided the environment primarily into two large categories: historic and discontinuous. In historic environments, decisions about the future are based on past and present events that can be extrapolated into the future. Change is incremental, predictable, and visible. In discontinuous environments, the future is partially visible and predictable; therefore, change is possible by using weak signals from the environment. Lastly, the future could be completely unpredictable and invisible; hence, changes are based on building scenarios utilizing weak environmental signals (Emery and Trist, 1965).

Scenario building using weak environmental signals as well as planning for discontinuous environments is the natural operating environment for a small business SB and is why Ansoff's theories may be relevant to the analysis of a SB. Although Ansoff's theories have been empirically validated for large firms, very little research has been done on their applicability to small business (Kipley, 2009). This analysis will use selected Ansoff theories and the OSPP models to determine SB descriptive and prescriptive outlook as well as ability to ensure Anasoff's Strategic Success Paradigm (SSP) in alignment. Alignment is complete when the SSP can declare three things:

“[1.] the aggressiveness of the firm's strategic behavior must match the turbulence of its environment, [2.] the responsiveness of the firm's capabilities must match the aggressiveness of its strategy and [3] the components of the firm's capabilities must be supportive of one another” (Ansoff, 2007). 


\section{Macrothink}

Journal of Management Research

ISSN 1941-899X

2013, Vol. 5, No. 2

The SSP uses the strategic gap analysis SGA to assess strategic effectiveness. SGA is a technique in which the difference between the desired performance levels and the extrapolated of the present performance levels is measured and examined. This will indicate should be done and what resources are required to meet its goals

An analysis of the strategic gaps" of SB will be conducted and reported; this analysis shows any disparity in the alignment between a SB's current and desired positions with respect to environmental turbulence, strategic aggressiveness and management responsiveness. Ansoff stipulated, "as the strategic gap increases, performance of the business in highly intense competitive environments declines more rapidly than performance of a business in a less intense competitive environment” (Ansoff, 1984).

Kipley and Lewis' Optimal Strategic Performance Position (OSPP) tool was used to assess the firm's strategic gaps with the goal of improving the match between the firm's operating environment and its current level of strategic aggressiveness and organization capability. The OSPP specifically measures the alignment between environmental turbulence, strategic aggressiveness and management responsiveness of a business in essence testing Ansoff's SSP. (Figure 1) depicts Ansoff's environment turbulence matrix.

\begin{tabular}{|c|c|c|c|c|c|}
\hline TURBULENCE LEVEL & 1 & 2 & 3 & 4 & 5 \\
\hline $\begin{array}{l}\text { Environmental Turbulence } \\
\text { (includes: available resources, } \\
\text { market demand, competitors, } \\
\text { regulatory frameworks, socio- } \\
\text { political climate) }\end{array}$ & REPETITIVE & EXPANDING & $\begin{array}{l}\text { CHANGING } \\
\text { Planned } \\
\text { Obsolescence }\end{array}$ & $\begin{array}{l}\text { DISCONTINUOUS } \\
\text { Unplanned } \\
\text { Obsolescence }\end{array}$ & $\begin{array}{l}\text { SURPRISEFUL } \\
\text { System Shock (e.g. } \\
\text { financial crisis) } \\
\text { Natural Disaster } \\
\text { Disruptive } \\
\text { Innovation/ } \\
\text { Unforseen New } \\
\text { Player (e.g. } \\
\text { competitor } \\
\text { announces } \\
\text { unforeseen \& } \\
\text { gamechanging } \\
\text { breakthrough) }\end{array}$ \\
\hline Levels of capability: & CUSTODIAL & PRODUCTION & MARKETING & STRATEGIC & FLEXIBLE \\
\hline TYPE OF RESPONSE & & TIVE & & PROACTIVE & \\
\hline Strategic Aggressiveness & STABLE & REACTIVE & ANTICIPATORY & ENTREPRENEURIAL & CREATIVE \\
\hline Management Responsiveness & $\begin{array}{l}\text { STABILITY } \\
\text { SEEKING }\end{array}$ & $\begin{array}{l}\text { EFFICIENCY } \\
\text { DRIVEN }\end{array}$ & MARKET DRIVEN & $\begin{array}{l}\text { ENVIRONMENT } \\
\text { DRIVEN }\end{array}$ & $\begin{array}{l}\text { ENVIRONMEN } \\
\text { T CREATING }\end{array}$ \\
\hline Market/Product Position & $\begin{array}{l}\text { MARKET } \\
\text { PENETRATION } \\
\text { (Existing Products } \\
\text { w/ Existing } \\
\text { Markets) }\end{array}$ & $\begin{array}{l}\text { PRODUCT } \\
\text { DEVELOPMENT } \\
\text { (New Products w/ } \\
\text { Existing Markets) }\end{array}$ & $\begin{array}{l}\text { MARKET } \\
\text { DEVELOPMENT } \\
\text { (Existing Products w/ } \\
\text { New Markets) }\end{array}$ & $\begin{array}{l}\text { DIVERSIFICATION } \\
\text { (New Products w/ New } \\
\text { Markets) }\end{array}$ & $\begin{array}{l}\text { UNKNOWN } \\
\text { Hybrid Strategy }\end{array}$ \\
\hline
\end{tabular}

Figure 1. Compiled Ansoff Matrix (Created and adapted from various sources) 
Environmental Turbulence is the complexity, rapidity and predictability of change in the business environment. Strategic Aggressiveness is defined as the discontinuity and novelty of strategies and speed at which they are developed and implemented. Management Capability/Responsiveness is defined as the characteristics of the organization that give it the ability to support its strategies and respond to changes in the business environment.

At a turbulence level of 1 , there is virtually no change in the business environment. The pace of change at two is relatively slow and businesses can easily keep up with change. The pace of change at level three is comparatively fast (i.e., businesses must react quickly to keep up with changes). It is important to note that at turbulence levels two and three, changes occur but are largely predictable. When a business is at a turbulence level of four, some of the external changes are irregular or are not predictable from previous changes. The highest turbulence level is five; at this level, changes occur quickly, often, and sometimes unexpectedly. Successful organizations anticipate each of the levels of change.

For a small business, it is sensible that once the plan is implemented, employees have guidance for carrying out the plan and are not thrown off course many times for different reasons. An old saying from an unknown author says, "no plan survives first contact." To prepare for a level 4-5 environment, a business should develop plans as well as contingencies so that (a) when the business experiences outside turbulence, a business need not take its time to form a basic strategy, or, (b) the business is better positioned to change the turbulence level to its advantage.

When environmental turbulence occurs, if one has a strategic plan in place, reactions are quicker, and it is more likely that the business will not fall into a deep reactionary state which would typically be seen in a level 4 or 5 environment. This augments the entrepreneur or manager's ability to think strategically and react creatively. It is important to remember that uncertainty in all strategy "is a necessary element brought on by the intelligent and resourceful opposition” (Cleary, 1988).

\section{Environmental Turbulence Level ETL OSPP Analysis}

A SB strategic gap analysis was simulated using the Optimal Strategic Performance Positioning (OSPP) software tool. (Kipley \& Lewis, 2011, 2012).

OSPP measures a business with respect to Strategic Readiness, Budget, Future Competitive Position and Future Prospects. Ansoff's assessments for strategic aggressiveness, environmental turbulence, management responsiveness, culture, innovation, marketing, and technical capacity are incorporated in the model. This analysis is limited to environmental turbulence, strategic aggressiveness and management responsiveness.

Ansoff's first rule of the strategic success paradigm is: “the aggressiveness of the firm's strategic behavior must match the turbulence of its environment” (Ansoff, 1979). The analysis examined the start-up firm's aggressiveness characterized by the level of novelty, riskiness of strategies, and creativity; these factors were assessed in relation to the firm's current environment. To optimize the small business' performance and its level of strategic 


\section{Ml Macrothink}

Journal of Management Research

ISSN 1941-899X

2013, Vol. 5, No. 2

aggressiveness, this examination compared the level of turbulence. Refer to (Figure 2-9) simulation of analysis.

(ETL) Environment turbulence level assess on OSPP assessed a Future industry innovations turbulence of 2.00

\begin{tabular}{|c|c|c|c|c|c|c|}
\hline $\begin{array}{l}\text { Enviroamental Turbulence Level } \\
\text { Assessment (1-5) }\end{array}$ & & & & & & Enter Number \\
\hline Industry & 1 & 2 & 3 & 4 & 5 & \\
\hline $\begin{array}{l}\text { 1. Frequency of New Products In } \\
\text { Industry }\end{array}$ & \begin{tabular}{|c|}
$\begin{array}{c}\text { Infrequent } 5 \text { or } \\
\text { more yrs }\end{array}$ \\
\end{tabular} & Law & Moderate & High & Very High -Several per yr & 1 \\
\hline $\begin{array}{l}\text { 2. Length of Product life Cycle in } \\
\text { industry }\end{array}$ & \begin{tabular}{|c|}
$\begin{array}{c}\text { Very long } 10 \text { or } \\
\text { more yrs }\end{array}$ \\
\end{tabular} & Long $(7-10)$ & Moderate (3-7) & Short (1-3) & Short - less than 1yr & 1 \\
\hline $\begin{array}{l}\text { 3. Number of Competing } \\
\text { Technologies in industry }\end{array}$ & None & 1 & $2>3$ & $4>5$ & $5+$ & 1 \\
\hline 4. Industry Technological Intensity & Low & \begin{tabular}{|l|} 
Low increasing \\
\end{tabular} & Moderate & High & Very Hight & 5 \\
\hline 5. Rate of Technological Obsolescence & Low & Low & High & High & Very High & 1 \\
\hline $\begin{array}{l}\text { 6. Level of Product Performance } \\
\text { Differentiation in industry }\end{array}$ & None & Low & Moderate & High & $\begin{array}{c}\text { Drastic (based on } \\
\text { Discontinuous Technolegy) }\end{array}$ & 4 \\
\hline 7. Industry Societal Pressures & None & Moderate & Strong & Very Strong & Strong and Novel & 1 \\
\hline $\begin{array}{l}\text { 8. Visibility of future change events in } \\
\text { iadustry }\end{array}$ & $\begin{array}{l}\text { Complete } \\
\text { visibility }\end{array}$ & $\begin{array}{r}\text { Future visibility } \\
\text { is extrapelative }\end{array}$ & $\begin{array}{l}\text { Future visibility is } \\
\text { predictable }\end{array}$ & $\begin{array}{c}\text { Future visibility is } \\
\text { partially } \\
\text { predictable }\end{array}$ & $\begin{array}{c}\text { Future visibility is completely } \\
\text { unpredietable }\end{array}$ & 3 \\
\hline $\begin{array}{l}\text { 9. Industry's Demand for Growth } \\
\text { Capital }\end{array}$ & Low & Moderate & High & Very High & Very Illiqh & 1 \\
\hline $\begin{array}{l}\text { 10. Rate of Change in Technology in } \\
\text { industry }\end{array}$ & Very slow & Slow & Fast & $\begin{array}{c}\text { Discontinuous } \\
\text { Familiar }\end{array}$ & $\begin{array}{l}\text { Discontinuous } \\
\text { Novel }\end{array}$ & 1 \\
\hline \multirow[t]{2}{*}{$\begin{array}{l}\text { 11. Barriers to Entry of New } \\
\text { Competitors in industry }\end{array}$} & None & Low & Moderate & High & Very High & 3 \\
\hline & & & & & $\begin{array}{l}\text { Future Industry Innovation } \\
\text { Turbulence (2.) }\end{array}$ & 2.00 \\
\hline
\end{tabular}

Figure 2. Environmental Turbulence Level

\section{$\underline{\text { Strategic Aggressiveness }}$}

\section{$\underline{\text { Strategic Aggressiveness OSPP Analysis }}$}

(SAA) Strategic aggressiveness: Present strategic aggressiveness is 2.02 with Innovation gap of 0.41 , a marketing component gap of .01. With an overall strategic aggressiveness leaves the SB with a gap of .21

\begin{tabular}{|l|c|c|c|}
\hline \multicolumn{2}{|c|}{ STRATEGIC COMPONENTS } & $\begin{array}{c}\text { COMPONENT } \\
\text { GAPS }\end{array}$ & $\begin{array}{c}\text { CLOSING } \\
\text { COSTS }\end{array}$ \\
\hline INNOVATION AGGRESSIVENESS & 1.82 & 0.41 & \\
\cline { 3 - 4 } MARKETING AGGRESSIVENESS & 2.22 & 0.01 & \\
\hline FIRM'S AGGRESSIVENESS LEVEL & 2.02 & \\
\hline STRATEGIC AGGRESSIVENESS GAP & & 0.21 \\
\hline
\end{tabular}

Figure 3. OSPP Strategic Components

\section{Management Responsiveness/Capabilities}

The capabilities analysis focused on six areas: managers, culture, structure, systems, technology and capacity. The goal here is to align general management capabilities to industry environment.

According to Kipley, Managerial capabilities are those capabilities which align the essential personal managerial drive, such as: skills and abilities, knowledge of the (industry and other), 


\section{Macrothink}

Journal of Management Research

ISSN 1941-899X

2013, Vol. 5, No. 2

cognitive problem solving skills, leadership abilities, communication skills, propensity for risk, creativity, anticipatory, exploring, and entrepreneurial instincts (Kipley, 2009, p13).

\section{Capability Assessment OSPP Analysis}

(CCA) Capability component assessment: Present general manager responsiveness is 3.56 with a Gap of 1.33, culture 1.29: Gap 0.94, structure1.86/Gap .37, systems 1.71/Gap .51, technology 2.57/Gap .34 and capacity of 2/Gap .23. The firm's overall firm's responsiveness level is 2.16 with a capability responsiveness Gap of .06. (See Figures 4-9)

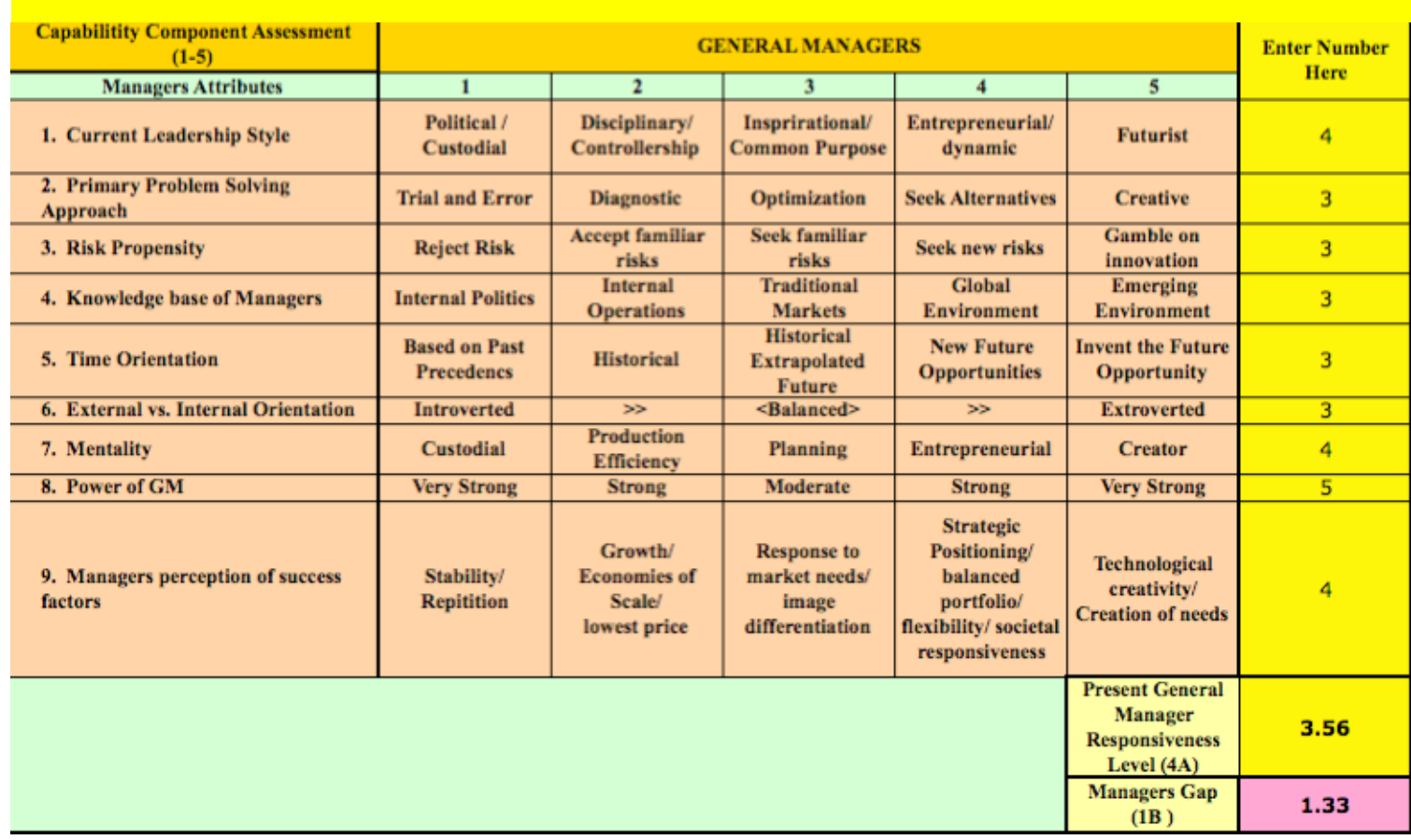

Figure 4. GM Assessment

\begin{tabular}{|c|c|c|c|c|c|c|}
\hline $\begin{array}{l}\text { Capabilitity Component Assessment } \\
\text { (1-5) }\end{array}$ & \multicolumn{5}{|c|}{ CULTURE } & \multirow{2}{*}{$\begin{array}{c}\text { Enter Number } \\
\text { Here }\end{array}$} \\
\hline Culture Attributes & 1 & 2 & 3 & 4 & 5 & \\
\hline 1. Current Rewards and Incentives & Length of service & Past Performance & $\begin{array}{l}\text { Contribution to } \\
\text { future growth }\end{array}$ & Entrepreneurship & Creativity & 2 \\
\hline 2. Values and Attitudes & Stability & Adaptation & Grow & Diversify & Create & 2 \\
\hline 3. Attitude toward Change & Reject & React & $\begin{array}{c}\text { Seek familiar } \\
\text { change }\end{array}$ & Seek Novel change & Create change & 3 \\
\hline 4. Propensity toward Risk Taking & Avoid & Only when forced & Tolerates & $\begin{array}{c}\text { Accepts Moderate } \\
\text { Risk }\end{array}$ & Accepts High Risk & 3 \\
\hline 5. What Triggers the need for Change & Crisis & $\begin{array}{c}\text { Accumulation of } \\
\text { Unsatisfactory } \\
\text { performance } \\
\end{array}$ & $\begin{array}{l}\text { Responding to } \\
\text { market }\end{array}$ & Seeking Change & Creating Change & 4 \\
\hline $\begin{array}{l}\text { 6. Time Perspective in which } \\
\text { Management percieves its problems }\end{array}$ & Past & $>$ & Present & $>$ & Future & 2 \\
\hline \multirow[t]{3}{*}{ 7. Success Criterion } & Stability & $\begin{array}{l}\text { Efficiency/ } \\
\text { Performance }\end{array}$ & $\begin{array}{c}\text { Effective } \\
\text { Response to } \\
\text { competition and } \\
\text { market needs } \\
\end{array}$ & $\begin{array}{l}\text { Dynamic balance of } \\
\text { the organization } \\
\text { portfolio/ Growth }\end{array}$ & $\begin{array}{l}\text { Innovation } \\
\text { leadership }\end{array}$ & 2 \\
\hline & & & & & $\begin{array}{c}\text { Present Culture } \\
\text { Responsiveness } \\
\text { Level (4B) } \\
\end{array}$ & 2.57 \\
\hline & & & & & Culture Gap (1B) & 0.81 \\
\hline
\end{tabular}

Figure 5. Culture Assessment 


\section{Macrothink}

Journal of Management Research

ISSN 1941-899X

2013, Vol. 5, No. 2

\begin{tabular}{|c|c|c|c|c|c|c|}
\hline $\begin{array}{c}\text { Capabilitity Component Assessment } \\
(1-5)\end{array}$ & \multicolumn{5}{|c|}{ STRUCTURE } & \multirow{2}{*}{$\begin{array}{c}\text { Enter Number } \\
\text { Here }\end{array}$} \\
\hline Structure Attributes & 1 & 2 & 3 & 4 & 5 & \\
\hline 1. Current Organizational Form & Bureaucratic & Functional & Divisional & $\begin{array}{l}\text { Matrix/ New } \\
\text { Venture }\end{array}$ & $\begin{array}{l}\text { Flexible } \\
\text { structure }\end{array}$ & 2 \\
\hline 2. Organizational structure focus & Specific task & Performance & Organic Growth & $\begin{array}{c}\text { Industry } \\
\text { Opportunity }\end{array}$ & $\begin{array}{l}\text { Industry } \\
\text { Growth }\end{array}$ & 2 \\
\hline $\begin{array}{l}\text { 3. Organizationa Structural } \\
\text { Flexibility }\end{array}$ & Rigid & Low Flexibility & $\begin{array}{l}\text { Moderate } \\
\text { Flexibility }\end{array}$ & Adaptive & Highly Adaptive & 2 \\
\hline 4. Current System & Control & Budgeting & LRP & $\begin{array}{l}\text { Strategic } \\
\text { Planning }\end{array}$ & $\begin{array}{c}\text { Issue/Surprise } \\
\text { Management }\end{array}$ & 2 \\
\hline 5. Management Focus & $\begin{array}{l}\text { Control of } \\
\text { deviation }\end{array}$ & $\begin{array}{l}\text { Allocation of } \\
\text { resources }\end{array}$ & $\begin{array}{l}\text { Coordination of } \\
\text { growth/profits }\end{array}$ & $\begin{array}{l}\text { Management of } \\
\text { Strategic } \\
\text { Innovation }\end{array}$ & $\begin{array}{c}\text { Management of } \\
\text { Partially/ } \\
\text { unpredictable } \\
\text { change }\end{array}$ & 2 \\
\hline 6. Primary purpose of structure & $\begin{array}{c}\text { Maintain status } \\
\text { quo }\end{array}$ & $\begin{array}{l}\text { Minimize } \\
\text { operating costs } \\
\text { of the firm }\end{array}$ & $\begin{array}{l}\text { Optimize the } \\
\text { firm's profits }\end{array}$ & $\begin{array}{c}\text { Develop the } \\
\text { firm's near term } \\
\text { profit potential }\end{array}$ & $\begin{array}{c}\text { Develop the } \\
\text { firm's long term } \\
\text { profit potential }\end{array}$ & 2 \\
\hline $\begin{array}{l}\text { 7. Power Center within the } \\
\text { organization }\end{array}$ & Bureaucratic & Production & Marketing & $\begin{array}{c}\text { General } \\
\text { Management }\end{array}$ & $\begin{array}{c}\text { Research \& } \\
\text { Development }\end{array}$ & 1 \\
\hline & & & & & $\begin{array}{c}\text { Present } \\
\text { Structure } \\
\text { Responsiveness } \\
\text { Level (4C) }\end{array}$ & 1.86 \\
\hline & & & & & $\begin{array}{l}\text { Structure Gap } \\
\text { (1B ) }\end{array}$ & 0.37 \\
\hline
\end{tabular}

Figure 6. Structure Assessment

\begin{tabular}{|c|c|c|c|c|c|c|}
\hline $\begin{array}{c}\text { Capabilitity Component Assessment } \\
(1-5)\end{array}$ & \multicolumn{5}{|c|}{ SYSTEMS } & \multirow{2}{*}{$\begin{array}{c}\text { Enter Number } \\
\text { Here }\end{array}$} \\
\hline Systems Attributes & 1 & 2 & 3 & 4 & 5 & \\
\hline $\begin{array}{l}\text { 1. Current Information gathering } \\
\text { system }\end{array}$ & Precedent based & Historical success & $\begin{array}{l}\text { Extrapolated } \\
\text { Future }\end{array}$ & \begin{tabular}{|c|} 
Scenario \\
Planning/ 'what-if \\
scenarios'
\end{tabular} & \begin{tabular}{|c|} 
Artificial \\
Intellegence/Data \\
Mining
\end{tabular} & 2 \\
\hline $\begin{array}{l}\text { 2. Current purpose priority of } \\
\text { systems within organization }\end{array}$ & Status Quo & Performance & Growth & \begin{tabular}{c|} 
New \\
Opportunities
\end{tabular} & Creativity & 1 \\
\hline 3. Organizations problem 'trigger' & React to crisis & \begin{tabular}{|c|}
$\begin{array}{c}\text { Accumulation of } \\
\text { unsatisfactory } \\
\text { performance }\end{array}$ \\
\end{tabular} & $\begin{array}{l}\text { Anticipated } \\
\text { threats }\end{array}$ & $\begin{array}{c}\text { New } \\
\text { Opportunities }\end{array}$ & Breakthroughs & 2 \\
\hline $\begin{array}{l}\text { 4. Organizations system for decision } \\
\text { making strategy }\end{array}$ & $\begin{array}{l}\text { Systems \& } \\
\text { Proceedures }\end{array}$ & Budgeting & $\begin{array}{c}\text { Extrapolative } \\
\text { strategic planning }\end{array}$ & $\begin{array}{c}\text { Entrepreneurial } \\
\text { strategic planning/ } \\
\text { capability } \\
\text { planning }\end{array}$ & $\begin{array}{c}\text { Strategic Issue } \\
\text { management / } \\
\text { Crisis } \\
\text { management }\end{array}$ & 2 \\
\hline 5. Current Systems typical problems & $\begin{array}{l}\text { Control of } \\
\text { Deviation }\end{array}$ & $\begin{array}{l}\text { Resource } \\
\text { allocation }\end{array}$ & $\begin{array}{l}\text { Coordinating } \\
\text { growth/profits }\end{array}$ & $\begin{array}{l}\text { Strategic } \\
\text { Coherence }\end{array}$ & $\begin{array}{c}\text { Management of } \\
\text { discontinuous } \\
\text { changes }\end{array}$ & 2 \\
\hline $\begin{array}{l}\text { 6. Procedures for Systems } \\
\text { Improvement }\end{array}$ & None & $\begin{array}{l}\text { When forced by } \\
\text { competition }\end{array}$ & $\begin{array}{l}\text { Accommodate } \\
\text { current growth }\end{array}$ & \begin{tabular}{|c|}
$\begin{array}{c}\text { Planned for } \\
\text { Future Capability } \\
\text { Needs }\end{array}$ \\
\end{tabular} & \begin{tabular}{|c|} 
Planned for \\
Future Creative \\
Capability Needs \\
\end{tabular} & 1 \\
\hline \multirow[t]{3}{*}{$\begin{array}{l}\text { 7. Which phrase best describes the } \\
\text { organization when control is lost }\end{array}$} & $\begin{array}{c}\text { Diviation from } \\
\text { stable state }\end{array}$ & $\begin{array}{c}\text { Deviation from } \\
\text { budgets }\end{array}$ & $\begin{array}{c}\text { Deviation from } \\
\text { plans }\end{array}$ & $\begin{array}{c}\text { Seek new } \\
\text { opportunities }\end{array}$ & Creative drive & 2 \\
\hline & & & & & $\begin{array}{c}\text { Present Systems } \\
\text { Responsiveness } \\
\text { Level (4D) } \\
\end{array}$ & 1.71 \\
\hline & & & & & Systems Gap (1B) & 0.51 \\
\hline
\end{tabular}

Figure 7. Systems Assessment 


\section{Ml Macrothink}

Journal of Management Research

ISSN 1941-899X

2013, Vol. 5, No. 2

\begin{tabular}{|c|c|c|c|c|c|c|}
\hline Capabilitity Component Assessment & \multicolumn{5}{|c|}{ MANAGEMENT TECHNOLOGY } & \multirow{2}{*}{$\begin{array}{c}\text { Enter Number } \\
\text { Here }\end{array}$} \\
\hline Management Technology Attributes & 1 & 2 & 3 & 4 & 5 & \\
\hline $\begin{array}{l}\text { 1. Current Analytical Model being } \\
\text { used by organization }\end{array}$ & $\begin{array}{l}\text { Standard } \\
\text { proceedures }\end{array}$ & $\begin{array}{c}\text { Work study/ Ratio } \\
\text { analysis/ } \\
\text { Equipment } \\
\text { replacement }\end{array}$ & $\begin{array}{c}\text { Capital budgeting/ } \\
\text { Optimization }\end{array}$ & Futurology & Creativity & 3 \\
\hline 2. Process Technology Level & None & Low & Moderate & Advanced & Industry Leader & 3 \\
\hline 3. Technology Acquisition & Minimal & Reactive & Proactive & $\begin{array}{l}\text { Opportunity } \\
\text { Seeking }\end{array}$ & $\begin{array}{c}\text { Opportunity } \\
\text { Creating }\end{array}$ & 2 \\
\hline 4. Product/Service Innovation & None & Low & Moderate & High & Industry Leader & 3 \\
\hline 5. Investment in Technology & None & Low & Moderate & High & Industry Leader & 3 \\
\hline 6. Technological positioning & Imitator & $>$ & Follower & $>$ & Innovator & 3 \\
\hline \multirow[t]{3}{*}{$\begin{array}{l}\text { 7. Current Technological } \\
\text { Surveillance system being used by } \\
\text { organization }\end{array}$} & Statistical Files & $\begin{array}{c}\text { Statistical } \\
\text { performance } \\
\text { control }\end{array}$ & $\begin{array}{l}\text { Performance } \\
\text { Extrapolation }\end{array}$ & \begin{tabular}{|c|} 
Non-linear \\
forecasting/ what- \\
if models
\end{tabular} & $\begin{array}{c}\text { Artificial } \\
\text { Intellegence }\end{array}$ & 1 \\
\hline & & & & \multicolumn{2}{|c|}{ Present Management Technology } & 2.57 \\
\hline & & & & & $\begin{array}{l}\text { Teehnology Gap } \\
\text { (1B) }\end{array}$ & 0.34 \\
\hline $\begin{array}{l}\text { Capabilitity Component Assessment } \\
\qquad(1-5)\end{array}$ & \multicolumn{5}{|c|}{ MANAGEMENT CAPACITY } & Enter Number \\
\hline Management Capacity Attributes & 1 & 2 & 3 & 4 & 5 & \\
\hline $\begin{array}{l}\text { 1. Which term best describes the } \\
\text { capacity of your organizaiton's } \\
\text { general management }\end{array}$ & Minimal & $\begin{array}{l}\text { Adequate for } \\
\text { breakeven }\end{array}$ & $\begin{array}{l}\text { Sufficient for } \\
\text { profit making } \\
\text { work }\end{array}$ & $\begin{array}{c}\text { Sufficient for } \\
\text { strategic and } \\
\text { profit making } \\
\text { work }\end{array}$ & $\begin{array}{c}\text { Sufficient for } \\
\text { creativity/ } \\
\text { strategic/ and } \\
\text { profit making } \\
\text { work }\end{array}$ & 2 \\
\hline \multirow[t]{4}{*}{$\begin{array}{l}\text { 2. Which term best describes the } \\
\text { capacity of your organizations's staff }\end{array}$} & Minimal & $\begin{array}{l}\text { Adequate for } \\
\text { breakeven }\end{array}$ & $\begin{array}{l}\text { Sufficient for } \\
\text { profit making } \\
\text { work }\end{array}$ & $\begin{array}{c}\text { Sufficient for } \\
\text { strategic and } \\
\text { profit making } \\
\text { work }\end{array}$ & $\begin{array}{c}\text { Sufficient for } \\
\text { creativity/ } \\
\text { strategic/ and } \\
\text { profit making } \\
\text { work }\end{array}$ & 2 \\
\hline & & & & \multicolumn{2}{|c|}{ Present Management Capacity } & 2.00 \\
\hline & & & & & $\begin{array}{l}\text { Technology Gap } \\
\text { (1B) }\end{array}$ & 0.23 \\
\hline & & & \multicolumn{3}{|c|}{ Capabilty Responsiveness Gap (1B) } & 0.286 \\
\hline
\end{tabular}

Figure 8. Technology \& Capacity Assessment

\begin{tabular}{|l|c|c|c|}
\hline \multicolumn{2}{|c|}{ GENERAL MANAGEMENT CAPABILITY COMPONENTS } & COMPONENT & CLOSING \\
GAPS & COSTS \\
\hline MANAGERS & 3.56 & 1.33 & \\
\cline { 1 - 1 } \cline { 4 - 5 } CULTURE & 1.29 & 0.94 & \\
\cline { 1 - 1 } STRUCTURE & 1.86 & 0.37 & \\
\cline { 1 - 1 } SYSTEMS & 1.71 & 0.51 & \\
\cline { 1 - 1 } TECHNOLOGY & 2.57 & 0.34 & \\
\cline { 1 - 1 } CAPACITY & 2.00 & 0.23 & \\
\hline FIRM'S RESPONSIVENESS LEVEL & 2.16 & SUB TOTAL & \\
\hline CAPABILITY RESPONSIVENESS GAP & & COSTS & \\
\hline & & TOTAL COST & \\
\hline
\end{tabular}

Figure 9. OSPP output Matrix

Combined Strategic Aggressiveness and Management Capability Assessment

The OSPP gap analysis shows (Figures 4-9) the firm is decently aligned (i.e. small gap) with respect to strategic aggressiveness and marketing. However, present general manager capabilities show a suboptimal gap. Previous research has shown that a Strategic Gap in the vicinity of 1.5 to 2.0 performances declines to zero (Ansoff, Sullivan, et al., 1993, p. 194). In order to correct this problem the management must transform its capability so that it is better 


\section{Macrothink}

aligned with the current environment.

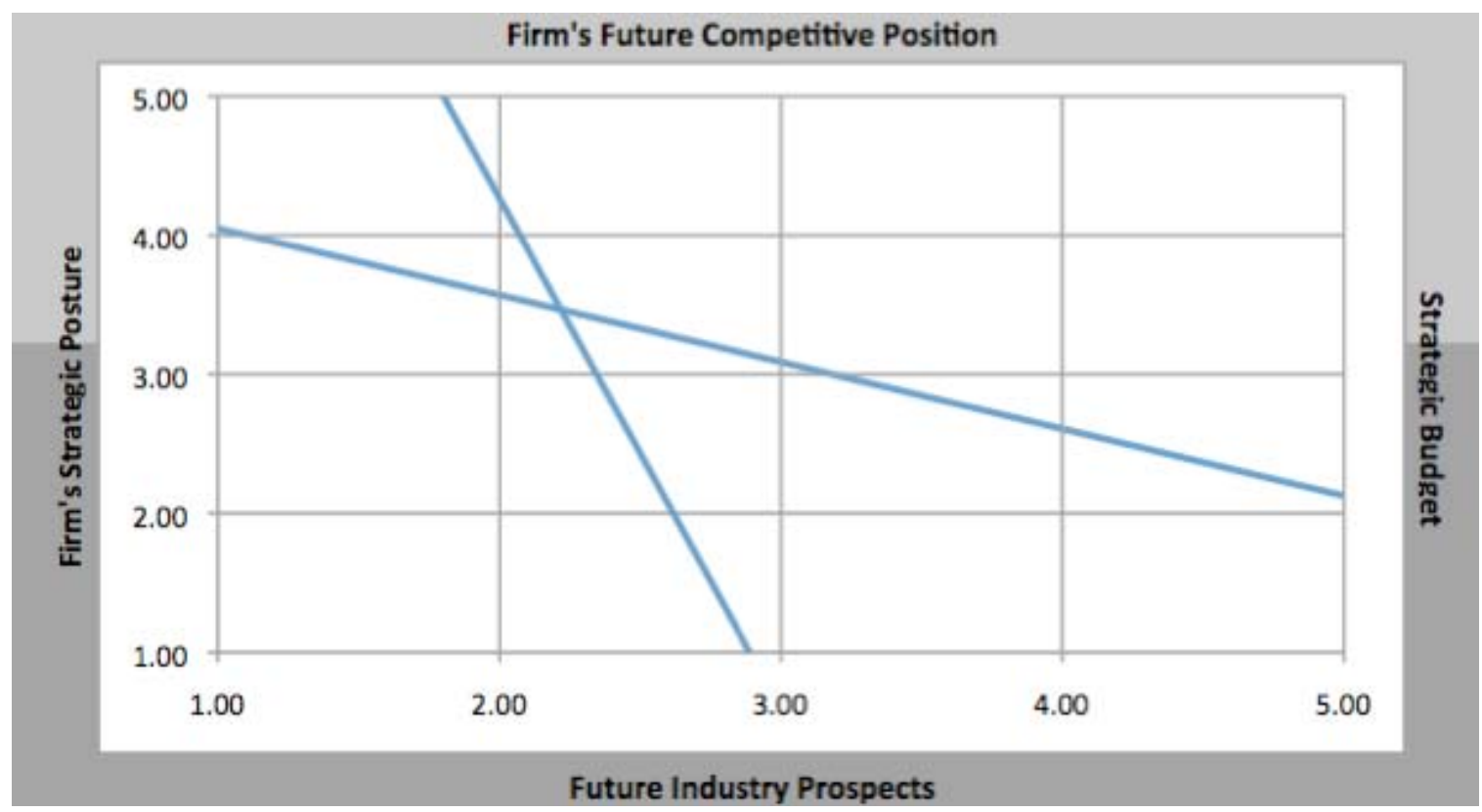

Figure 10. OSPP Matrix Alignment Graph

The matrix (Figure 10) shows a visual reference of where the SB posture is and where management can increase its strategic position. Strategic posture is aggressive while strategic budget can increase. Future industry prospects are relatively moderate while management may choose to increase the strategic budget to align more with the environment.

\section{Summary}

The purpose of the article was to give you a brief introduction to Igor Ansoff, his theories, and review the OSPP tool in accordance with the strategic success paradigm variables:

Ansoff theories have been backed by several empirical studies. We discussed Ansoff's Strategic Success Paradigm and principles for use by small business, which when implemented have proven empirically to increase the firm's probability of strategic success. There is also strong empirical support linking a positive causal relationship between formalized strategic planning and achieving optimal financial success of a business organization.

A SB theoretically can better position itself to be successful by following Ansoff's theories and incorporating and using the OSPP tool by aligning the business capabilities with the environment and thus providing both a descriptive plan as well as a prescriptive diagnosis to their strategic plan. Utilized along with knowledge, practical experience, other modeling techniques, using the OSPP tool, for can lead to a small business's successful strategy. Future research needs to further study Ansoffian theories, the OSPP and its effects on small business performance.

\section{References}

Ansoff, H.I. (1984). Implanting strategic management. Prentice Hall International, NY NY 
Ansoff, H.I., \& McDonnell, E. (1990), Implanting Strategic Management (2nd Edi.), Prentice-Hall, New York, NY.

Ansoff, H.I., \& Sullivan, P. A. et al. (1993). Empirical Proof of a Paradigmatic Theory of Strategic Success Behaviors of Environment Serving Organizations. International Review of Strategic Management, 4, 173-202.

Ansoff, H.I. (2007). Strategic Management Classic, Palgrave Macmillan. Hampshire, UK. http://dx.doi.org/10.1057/9780230590601

Ashby, W. R. (1958). Requisite variety and its implications for the control of complex systems. Cybernetica (Namur), 1(2), 83-99.

Cleary, T. (1988). The Art of War by Sun Tzu (Translated.). Boston, MA: Shambhala Publications Inc. Metrieved May 13, 2011, from http://www.amazon.com/Art-War-Sun-Tzu/dp/1590302257.

Consulting, E.-K. (2011). Igor Ansoff the father of Strategic management From A to Z Strategy Gurus. Retrieved May 11, from http://www.easy-strategy.com/igor-ansoff.html.

Emery, F. E., \& Trist, E. L. (1965). The Causal Texture of Organizational Environments. Human Relations, 18(1), 21-32. http://dx.doi.org/10.1177/001872676501800103

Ghyczy, T. von, Bassford, C., \& Oetinger, B. von. (2001). Clausewitz on Strategy: Inspiration and Insight from a Master Strategist. New York: John Wiley \& Sons, Inc.

Greenfield, W. M. successful management consulting: Building a practice with smaller company clients. Englewood Cliffs, NJ: Prentice-Hall

Hoover's. Inc. (2011). Education and Training Services Industry Profile from First Research. Retrieved May 11, 2011, from http://www.firstresearch.com/Industry-Research/Education-and-Training-Services.html.

IBISWorld. (2010). Sports Coaching Industry Research in the US by IBISWorld. Retrieved May 11, 2011, from http://www.ibisworld.com/industry/default.aspx?indid=1542.

Infogroup, I. (n.d.). ReferenceUSA. Retrieved May 11, 2011, from http://www.referenceusa.com/.

Kipley, D. H., \& Lewis, A. O. (2009). The Scalability of H. Igor Ansoff's Strategic Management Principles for Small and Medium Sized Firms. Journal of Management Research, 1(1), 1-26. Las Vegas: Macrothink Institute. Retrieved May 11, 2011, from http://www.macrothink.org/journal/index.php/jmr/article/view/E6.

Kipley, D. H., \& Lewis, A. O. (2009). A Tricotomic Examination of the Planning School, Learning School, and Positioning School Relative to Achieving Optimal Financial Performance in Discontinuous Environmental Turbulence Levels. Journal of Management Research, 1(2), 16. Retrieved May 11, 2011, from http://www.macrothink.org/journal/index.php/jmr/article/view/E11. 


\section{Macrothink}

Journal of Management Research

ISSN 1941-899X 2013, Vol. 5, No. 2

Kipley, D. H., \& Lewis, A. O. (2009). Optimal Strategic Performance Position (OSPP) Matrix [computer software].

Mintzberg, H. (1994). Fall and Rise of Strategic Planning - Harvard Business Review. Harvard Business Review, 107-114. Retrieved May 11, 2011, from http://hbr.org/product/fall-and-rise-of-strategic-planning/an/94107-PDF-ENG.

Mintzberg, H., Lampel, J., \& Ahlstrand, B. (1998). Strategy Safari: A Guided Tour Through The Wilds of Strategic Management. New York, NY: THE FREE PRESS.

Moussetis, R. (2011). How Ansoff interfaces with both the planning and learning schools of thought in strategy. Emerald Group Publishing. http://dx.doi.org/10.1108/17511341111099556

Porter, M. E. (1980). Competitive Strategy. New York: The Free Press.Rue \& Ibrahim, Pun, S. S. (n.d.). Managing in Turbulent Times: Igor Ansoff's Strategic Success Model. Retrieved from www.ansoffasia.com/article002.pdf.

Rivers, K. (2011, April). FUSD Holds Bullying Forum for Parents | The Fillmore Gazette. The Fillmore Gazette: Newspaper of Record for the City of Fillmore, Front Page. Fillmore. Retrieved May 13, 2011, from http://www.fillmoregazette.com/front-page/fusd-holds-bullying-forum-parents.

SCORE. (2011). Marketing Basics. Retrieved May 11, 2011, from http://www.score.org/resources/marketing-basics.

The Economist. (2008). Guru: Igor Ansoff. The Economist. Retrieved May 11, 2011, from http://www.economist.com/node/11701586.

Thompson, A. A., Strickland III, A. J., \& Gramble, J. E. (2005). Crafting and Executing Strategy (14th ed.). New York: McGraw-Hill/Irwin.

Tutor2u. (2011). strategy - competitive advantage. Retrieved May 11, from http://tutor2u.net/business/strategy/competitive_advantage.htm. 